\title{
Contamination Mitigation Strategy for Ultra-Low Energy Electron Microscopy and Spectroscopy
}

Eliška Materna Mikmeková ${ }^{*}$, Luděk Frank $^{1}$, Ivo Konvalina ${ }^{1}$, Ilona Müllerová ${ }^{1}$, Tao Zhang ${ }^{2}$ and Tewodros Asefa ${ }^{2}$

1. Department of Electron Microscopy, Institute of Scientific Instruments of the CAS, v. v. i., Brno, Czech Republic.

2. Department of Chemistry and Chemical Biology and Department of Chemical and Biochemical Engineering, Rutgers University, Piscataway NJ, USA.

* Corresponding author: eliska.mikmekova@isibrno.cz

Perhaps the most straightforward expectation related with ultra-low energy microscopy/spectroscopy is the low penetration of electrons into the samples. In general, low energy electrons cause more intense contamination than high energy electrons, which can be explained by diminution of the interaction volume of electrons inside the samples and increased yield of secondary electrons. Naturally, for a true "surface" study, the sample has to be perfectly clean and an in-situ cleaning method should be applied on the sample.

Even a small amount of hydrocarbon contamination can severely impact the results obtained with low energy electrons, as illustrated in Figure 1. During the scanning of surfaces by electrons, the image usually darkens because of a carbonaceous layer gradually deposited on the top from adsorbed hydrocarbon precursors. This effect can be described as electron stimulated deposition. The surface diffusion of hydrocarbon molecules around the irradiated area serves as a source of additional precursors, responsible for the even darker frame of the contaminated field of view. On the other hand, the effect of electron stimulated desorption occurs at the same time, especially at low energies, leading to a fundamental question as to what process, deposition or desorption, dominates. Beam irradiation at a high electron flux can also lead to surface cleaning. While irradiation by high energy electrons in the TEM practically sputters off the adsorbed species, energy below $1 \mathrm{keV}$ causes electron stimulated desorption based on multi-electron excitation processes. [1, 2] Unfortunately, the beam irradiation cleaning involves many free parameters, and yet only few papers have so far been published on this problem, especially involving low energy electrons - see Figure 2. [3, 4, 5]

The real "surface" studies of materials in microscopes equipped with selected spectroscopy techniques aren't common so far, which is due to the significant problem associated with the contamination. A range of specimen cleaning methods can be applied to selected samples. Typical cleaning methods, such as solvent rinsing, heating, ion bombardment and plasma etching, have their own limitations. Electroninduced in-situ cleaning procedure can be gentle, experimentally convenient and very effective for a wide range of specimens. Even already deposited contamination square on the surface can be removed by slow electrons, which is not possible by annealing in a vacuum. The annealing processes usually lead to the changes in the structure of deposited carbon-based film (contamination), e.g., typical graphite-like structure can be transformed into diamond-like carbon-based film. The results are illustrated in Figure 3 $[6]$.

References:

[1] RD Ramsier and JT Yates Jr., Surface Science Reports 12 (1991), p. 246. 
[2] TE Madye and JT Yates Jr., Journal of Vacuum Science and Technology 8 (1995), p. 525.

[3] L Frank, E Mikmeková and M Lejeune, Applied Surface Science 407 (2017), p. 105.

[4] E Mikmeková et al., Diamond and Related Materials 63 (2015), p. 136.

[5] S Suzuki in "Nanotechnology and Nanomaterials: Electronic Properties of Carbon Nanotubes", (2011) p. 329.

[6] The authors acknowledge funding from the Technology Agency of the Czech Republic (Centre of Electron and Photonic Optics, no: TN01000008 and TG03010046).

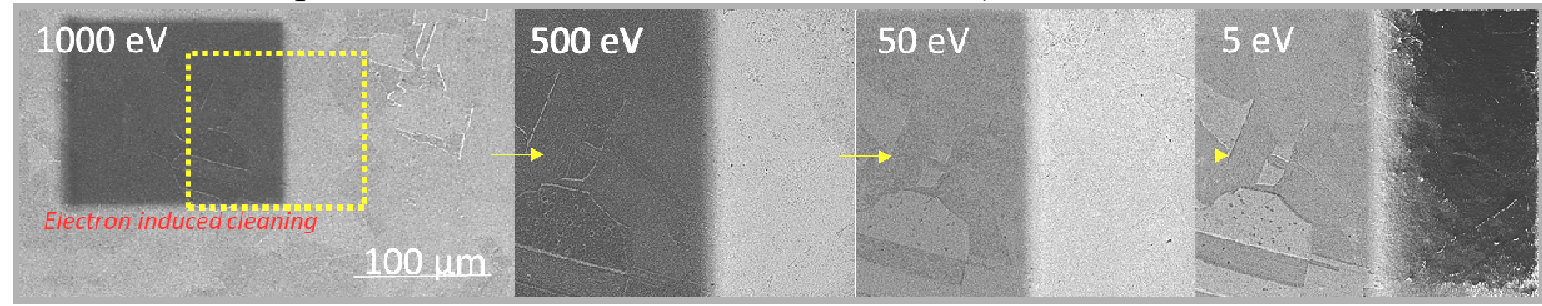

Figure 1. Metal-based foil observed with $1000 \mathrm{eV}, 500 \mathrm{eV}, 50 \mathrm{eV}$ and $5 \mathrm{eV}$ electrons (the right half of each frame shows the non-cleaned area covered with adsorbed molecules, whereas the left half shows the area cleaned by slow electrons, $500 \mathrm{eV}, 1 \mathrm{C} / \mathrm{cm}^{2}$ ).
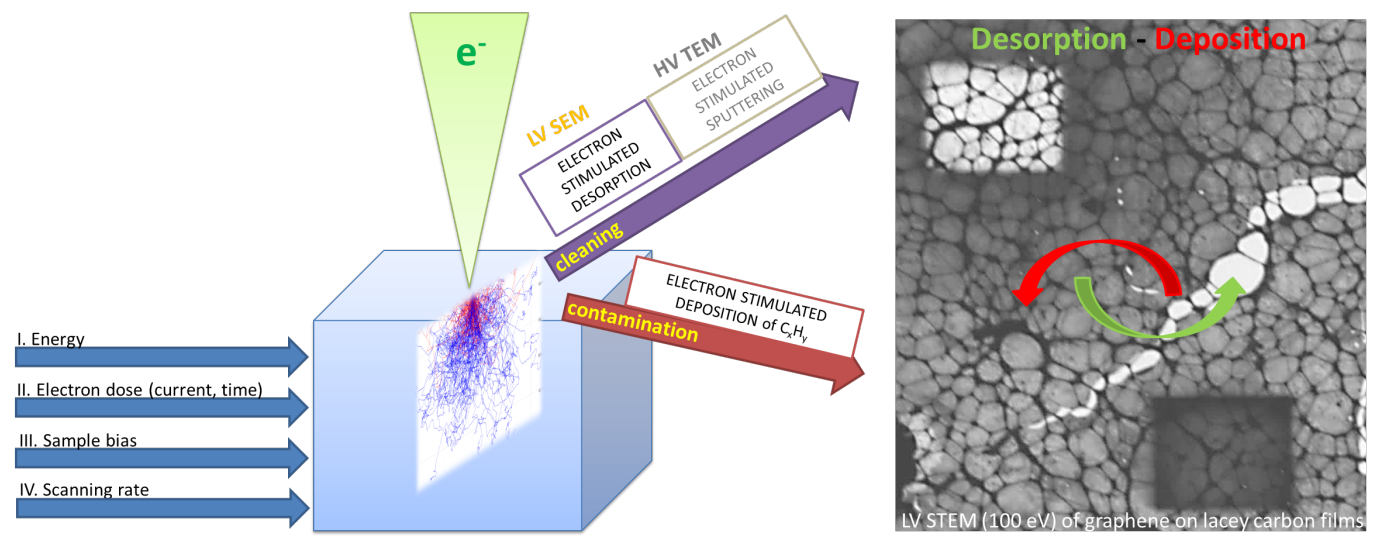

Figure 2. Parameters of electron beam and their effects on the deposition/desorption of hydrocarbons.

A.

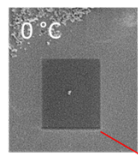

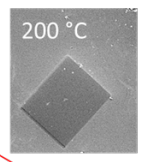
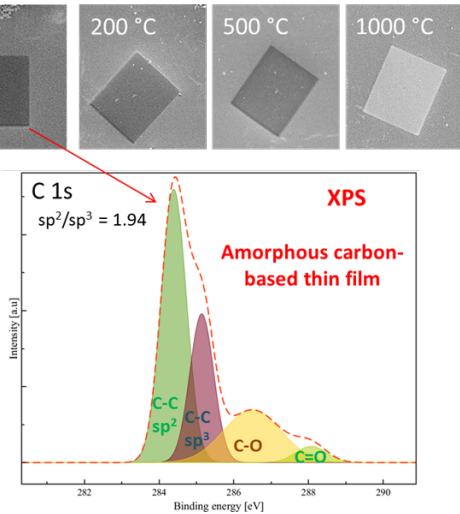

B.

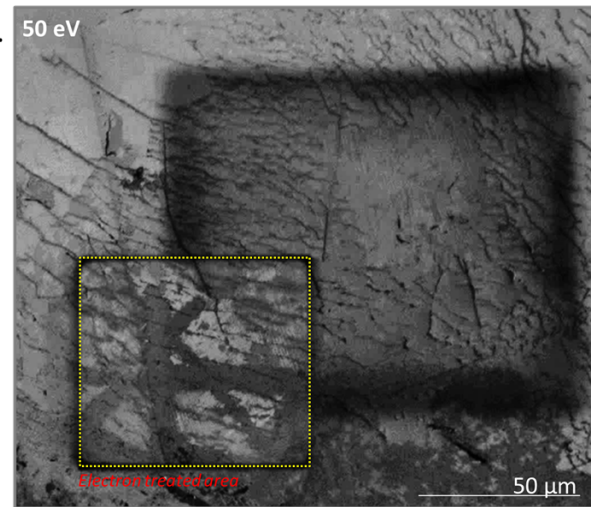

Figure 3. Removal of contamination square deposited by electron beam on copper foil: by annealing in vacuum (A.) and by slow electrons - $500 \mathrm{eV}$ (B.). 